\title{
Propriedades físico-mecânicas da madeira de Chrysophyllum marginatum
}

\author{
Jussan Albarello de Cezaro ${ }^{1 *}$, Rômulo Trevisan ${ }^{1}$, Rafaelo Balbinot ${ }^{1}$ \\ ${ }^{1}$ Universidade Federal de Santa Maria, Linha 7 de Setembro, CEP 98.400-000, Frederico Westphalen, RS, Brasil
}

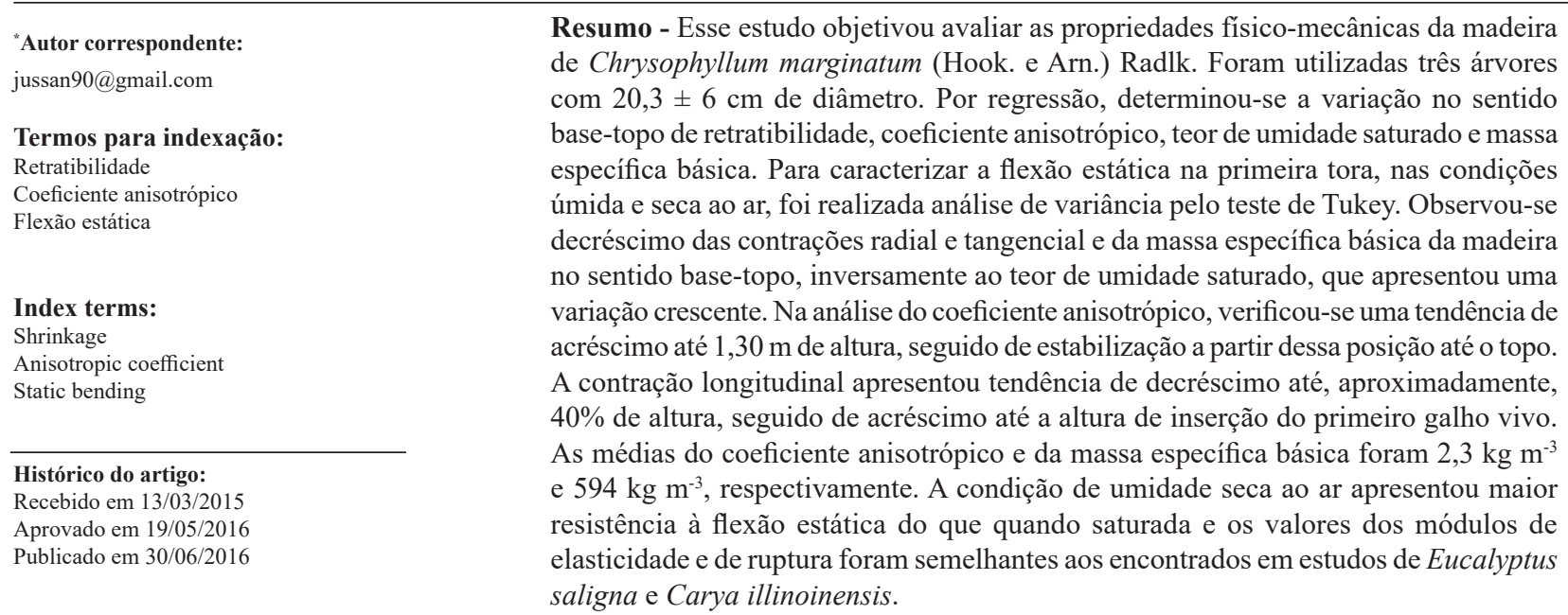

doi: $10.4336 / 2016 . p f b .36 .86 .884$

\section{Physical and mechanical properties of Chrysophyllum marginatum wood}

\begin{abstract}
This study aimed to evaluate the physical and mechanical properties of the wood of Chrysophyllum marginatum (Hook. And Arn.) Radlk. We used three trees with $20.3 \pm 6 \mathrm{~cm}$ of diameter. It was determined by regression analysis the variation bottomup of shrinkage, anisotropic coefficient, saturate moisture content and basic density. To characterize the static bending in the first log, in saturate and air dried conditions, it was performed variance analysis using Tukey>s test. It was observed a decrease in radial and tangential contractions and basic density on bottom-up direction. Saturate moisture content increased, considering the same direction. Anisotropic coefficient presented increase tendency up to $1.30 \mathrm{~m}$ height followed by stabilization from that position to the top. It was observed decreasing tendency of longitudinal contraction to approximately $40 \%$ of total height, followed by increasing up to the insertion of the first living branch. Mean anisotropic coefficient and basic density were $2.3 \mathrm{~kg} \mathrm{~m}^{-3}$ and $594 \mathrm{~kg} \mathrm{~m}^{-3}$, respectively. Air dry condition showed greater static bending resistance than when saturate. Values of rupture and elasticity modulus were similar to those found in studies with Eucalyptus saligna and Carya illinoinensis.
\end{abstract}

\section{Introdução}

$\mathrm{Na}$ avaliação da qualidade da madeira, a massa específica básica, a retratibilidade e a resistência aos esforços de flexão estática podem ser consideradas as características mais importantes dentre os parâmetros físico-mecânicos e seus valores expressam a combinação de diversos fatores, incluindo a constituição morfológica, anatômica e química do material (Tsoumis, 1991; Rezende, 2003; Calderazzo \& Yamaji, 2010). 
Por ser um material anisotrópico, a madeira pode alterar dimensionalmente e de forma desigual em relação aos planos de orientação, dependendo da umidade de equilíbrio do ambiente em que se encontra quando abaixo do PSF (ponto de saturação das fibras). A variação das propriedades físico-mecânicas também é diferente em relação aos planos anatômicos e deve ser avaliada para determinação da estabilidade dimensional do material (Oliveira, 1988; Durlo \& Marchiori, 1992; Oliveira \& Silva, 2003; Vicente et al., 2005). Esses autores afirmaram, ainda, que as variações dimensionais e a anisotropia são características indesejáveis da madeira, limitando o seu uso para diversas finalidades e exigindo, dessa maneira, técnicas específicas de processamento e utilização.

A massa específica básica também é um parâmetro importante para a caracterização da madeira, principalmente por estar relacionada com sua resistência e rigidez, variando em função da parede celular, dimensões, tipos de células e, em menor proporção, da quantidade de componentes extrativos presentes por unidade de volume (Panshin \& De Zeeuw, 1980; Haygreen \& Bowyer, 1982; Mattos et al., 2011).

A massa específica básica da madeira varia em função das características da própria espécie, bem como das influências externas, assim como das variações ambientais e intervenções silviculturais (Kollmann \& Côté, 1968; Brasil \& Ferreira, 1972; Rezende et al., 1995; Silva et al., 2004; Alzate et al., 2005; Roque, 2005; Trevisan et al., 2007). Além disso, alguns autores, como Panshin \& De Zeeuw (1980) e Lima et al. (2000), mencionam que essa característica aumenta muito rapidamente durante o período juvenil, cresce mais lentamente em um estágio intermediário da idade, até se tornar mais ou menos constante na fase da maturidade da árvore.

Outra propriedade de elevada importância para a determinação da qualidade da madeira, de acordo com Lopes \& Garcia (2002), é a umidade natural da madeira, ou seja, a umidade das árvores "vivas", expressa pela relação entre o peso da água contida na madeira recém cortada e o peso seco do material. Para os mesmos autores, é provável que essa tenha sido a primeira propriedade a ser efetivamente investigada e uma das mais importantes em todas as múltiplas utilizações da madeira, especificamente para construção civil e mobiliário.

Com relação às propriedades mecânicas, os módulos de ruptura (MOR) e de elasticidade (MOE) são dois parâmetros normalmente determinados nos testes de flexão estática e são importantes na caracterização tecnológica da madeira, pois ambos fornecem uma boa aproximação da resistência do material, constituindose, na prática, em critérios de classificação (Serpa et al., 2003).

Chrysophyllum marginatum (Hook. e Arn.) Radlk. pertence à família Sapotaceae e é denominado vulgarmente como aguaí, aguaí-leiteiro, aguaí-vermelho, entre outros. Com ocorrência na Bahia e, de Goiás até ao Rio Grande do Sul, principalmente na floresta latifoliada semidecídua de altitude e das bacias do Paraná e do Uruguai, é caracterizado como planta heliófila, seletiva higrófita, pioneira, característica e exclusiva de matas semidecíduas, onde ocorre com frequência por vezes abundante, porém bastante descontínua na sua dispersão (Lorenzi, 2002).

Os estudos sobre o aguaí-leiteiro ainda são escassos, fazendo com que não se conheça devidamente suas utilidades como madeira, embora acredita-se que possa ser utilizada para carpintaria em ambientes internos, móveis rústicos e reflorestamento com fins ecológicos. Portanto, o objetivo desse estudo foi avaliar as propriedades físico-mecânicas da madeira de $C$. marginatum (Hook. e Arn.) Radlk.

\section{Material e métodos}

\section{Caracterização da área e amostragem}

As amostras foram coletadas em área experimental da Universidade Federal de Santa Maria, Campus de Frederico Westphalen com, aproximadamente, 60 ha de floresta estacional decidual, sob coordenadas $27^{\circ} 23^{\prime} 46,68^{\prime \prime}$ S e $53^{\circ} 26^{\prime} 00,41^{\prime \prime} \mathrm{W}$. O clima da região é temperado mesotérmico brando super úmido sem seca, com temperatura média entre 10 e $15^{\circ} \mathrm{C}$, e os solos são caracterizados como Latossolos Roxos distróficos (Latossolos Vermelhos) (IBGE, 2014).

Foram selecionadas três árvores com diâmetro médio de $20,3 \pm 6 \mathrm{~cm}$. Para a avaliação das propriedades físicas foram retirados discos, mistos de cerne e alburno, com $12 \mathrm{~cm}$ de espessura, nas posições: 0,10 e $1,30 \mathrm{~m}$ de altura e nas posições 20, 40, 60, 80 e 100\% da altura de inserção do primeiro galho vivo. Para a determinação da flexão estática foram retirados toretes, mistos de cerne e alburno, com $50 \mathrm{~cm}$ de comprimento, entre 20 e $40 \%$ da altura de inserção do primeiro galho vivo. 


\section{Propriedades físicas}

Os discos foram acondicionados em tonel com água até massa constante e, após, foram marcados e seccionados em baguetas centrais (contendo a medula) de 2,5 $\mathrm{cm}$ de largura. Essas baguetas foram seccionadas na medula, sendo retirado $1 \mathrm{~cm}$ de cada lado da porção que continha a mesma, para que, posteriormente, fossem dimensionados os corpos de prova que seriam utilizados nos testes de retratibilidade, massa específica básica e teor de umidade saturado, seguindo a norma ASTM D143-94 (American Society for Testing Materials, 1995).

Foi realizada a marcação e numeração de 130 corpos de prova (38 da primeira árvore, 34 da segunda e 58 da terceira, em função da diferença de diâmetro existente), com dimensões de 2,5 x 2,5 x 10,0 cm (radial, tangencial e longitudinal, respectivamente), no sentido medulacasca. Então, procedeu-se com a mensuração das distâncias da medula até a casca, medula até o início do primeiro corpo de prova e distância do último corpo de prova até a casca para o cálculo da posição relativa. Em seguida, os corpos de prova foram seccionados e submersos em água onde permaneceram até massa constante.

Para o teste de retratibilidade, cada corpo de prova, inicialmente em estado verde, foi pesado em balança eletrônica digital com precisão de $0,01 \mathrm{~g}$ e mensurado na respectiva direção radial, tangencial e longitudinal. As dimensões nos planos radial e tangencial foram determinadas com espessímetro, com precisão de $0,01 \mathrm{~mm}$, em dois pontos previamente marcados nos corpos de prova. Na direção longitudinal, a mensuração foi realizada com paquímetro com precisão de $0,01 \mathrm{~mm}$.

Realizadas essas aferições, os corpos de prova foram então expostos à temperatura e umidade do ambiente até atingirem a umidade de equilíbrio. A partir dessa umidade, a secagem foi realizada em estufa com temperatura de $103{ }^{\circ} \mathrm{C}$, para determinação da massa seca e das dimensões radial, tangencial e longitudinal, na condição $0 \%$ de umidade. A contração nos três planos anatômicos e o coeficiente anisotrópico foram determinados, respectivamente, pelas expressões 1 e 2 .

$\beta_{\mathrm{tg}} ; \beta_{\mathrm{rd}} ; \beta_{\mathrm{lg}}=\left[\mathrm{D}_{\mathrm{u}}-\mathrm{D}_{\mathrm{o}}\right) / \mathrm{D}_{\mathrm{u}} * 100$

Em que: $\beta_{\mathrm{tg}} ; \beta_{\mathrm{rd}} ; \beta_{\mathrm{lg}}=$ contração tangencial, radial e longitudinal, respectivamente $(\%) ; \mathrm{D}_{\mathrm{u}}=$ dimensão na condição saturada (acima PSF) (m); $\mathrm{D}_{0}=$ dimensão na condição de $0 \%$ de umidade $(\mathrm{m})$.

$\mathrm{CA}=\beta_{\mathrm{tg}} / \beta_{\text {rd }}$

Em que: $\mathrm{CA}=$ coeficiente anisotrópico; $\beta_{\mathrm{tg}}=$ contração na direção tangencial $(\%) ; \beta_{\mathrm{rd}}=$ contração na direção radial (\%).
Para a determinação da massa específica básica, o volume saturado dos corpos de prova foi mensurado pelo método da balança hidrostática (Vital, 1984), e a massa seca foi obtida, pelo método gravimétrico, deixando-se as amostras em estufa a $103{ }^{\circ} \mathrm{C}$ até massa constante (Equação 3).

$\mathrm{MEB}=\mathrm{P}_{0 \%} / \mathrm{V}_{\mathrm{V}}$

Em que: $\mathrm{MEB}=$ massa específica básica $\left(\mathrm{kg} \mathrm{m}^{-3}\right) ; \mathrm{P}_{0 \%}=$ massa seca na condição de $0 \%$ de umidade $(\mathrm{kg}) ; \mathrm{V}_{\mathrm{v}}=$ volume saturado $\left(\mathrm{m}^{3}\right)$.

O teor de umidade saturado também foi determinado por meio do método gravimétrico onde se procedeu a mensuração da massa dos corpos de prova nas condições saturada e $0 \%$ de umidade (após secagem em estufa a $103^{\circ} \mathrm{C}$ até massa constante), sendo calculado conforme equação 4.

TUS $=\left[\left(\mathrm{P}_{\mathrm{U}}-\mathrm{P}_{\mathrm{S}}\right) / \mathrm{P}_{\mathrm{S}}\right] * 100$

Em que: TUS $=$ teor de umidade saturado $(\%) ; \mathrm{P}_{\mathrm{u}}=$ massa na condição saturada $(\mathrm{kg}) ; \mathrm{P}_{\mathrm{s}}=$ massa seca na condição de $0 \%$ de umidade $(\mathrm{kg})$.

\section{Flexão estática}

No ensaio de flexão estática também foi utilizada a norma ASTM D143-94 (American Society for Testing Materials, 1995), onde os toretes foram seccionados em pranchões e, posteriormente, dimensionados em 30 corpos de prova de $2,5 \mathrm{~cm}$ x 2,5 $\mathrm{cm}$ x 41,0 cm (radial, tangencial e longitudinal, respectivamente). $\mathrm{O}$ teste foi realizado em máquina universal de ensaios nas condições úmida, acima do PSF, e seca ao ar, próximo a $15 \%$ de teor de umidade, determinando as seguintes variáveis: força máxima $\left(\mathrm{F}_{\text {máx }}\right)$ em $\mathrm{N}$ e módulos de ruptura (MOR), e de elasticidade (MOE) e tensão no limite proporcional (TLP) em $\mathrm{N} \mathrm{m}^{-2}$.

\section{Análise dos dados}

Para a análise da variação no sentido base-topo da MEB, do CA, da $\beta$ (tangencial, radial e longitudinal) e do TUS, os dados amostrados por posição foram submetidos à análise de regressão, sendo processados pelo pacote Statistical Analysis System (Statistical Analysis System, 1993), no qual foi, primeiramente, aplicado o procedimento Stepwise de modelagem de regressão em função da posição relativa no sentido axial, por meio da expressão 5 .

MEB; CA; $\beta$ tg; $\beta r d ; \beta \lg ;$ TUS = f $\left(\mathrm{P} ; 1 / \mathrm{P} ; \mathrm{P}^{2} ; 1 / \mathrm{P}^{2} ; \operatorname{lnP} ; 1 /\right.$ $\ln \mathrm{P} ; \sqrt{ } \mathrm{P})$

Em que: $\mathrm{MEB}=$ massa específica básica $\left(\mathrm{kg} \mathrm{m}^{-3}\right) ; \mathrm{CA}=$ coeficiente anisotrópico; $\beta=$ contração na direção tangencial, radial e longitudinal, respectivamente (\%); TUS = teor de umidade saturado (\%); $\mathrm{P}=$ posição relativa no sentido axial $(\%)$

Pesq. flor. bras., Colombo, v. 36, n. 86, p. 135-143, abr./jun. 2016 
O melhor modelo foi selecionado com base no coeficiente de determinação ajustado $\left(\mathrm{R}^{2}{ }_{\mathrm{aj}}\right)$, erro padrão da estimativa $\left(\mathrm{S}_{\mathrm{xy}}\right)$, coeficiente de variação $(\mathrm{CV})$, valor de F calculado e análise da distribuição dos resíduos.

A flexão estática nas condições saturada e seca ao ar foi interpretada com auxílio de uma análise da variância (ANOVA) e, no caso de rejeição da hipótese de igualdade entre os tratamentos, pelo teste de comparação de médias de Tukey com probabilidade de erro fixado em 5\%.

\section{Resultados e discussão}

\section{Propriedades físicas}

As contrações tangencial, radial e longitudinal variaram de forma diferente nos três planos de orientação da madeira, tanto na intensidade quanto no sentido base-topo (Figura 1), corroborando com as afirmações de Bodig \& Jayne (1982); Burger \& Richter (1991) e Oliveira \& Silva (2003) que relataram a possibilidade de variação dessas características entre espécies, entre indivíduos de uma mesma espécie e até em uma mesma árvore, nos sentidos axial e transversal.

Pode ser observado na Figura 1 que a contração tangencial variou de 5,81 (topo) a 9,92\% (base), com $8,4 \%$ de média, apresentando tendência de decréscimo no sentido base-topo, com valores máximos próximos à altura de corte $(0,10 \mathrm{~m})$ e mínimos a $100 \%$ da altura de inserção do primeiro galho vivo. Da mesma forma, a contração radial também mostrou uma tendência de decréscimo no sentido base-topo, com valores entre 1,13 (topo) e 5,59\% (base) e 3,6\% de média. A contração longitudinal variou entre 0,06 ( $40 \%$ da altura de inserção do primeiro galho vivo) e $0,90 \%$ (topo), com média de $0,37 \%$, e tendência de decréscimo até, aproximadamente, $40 \%$ de altura, seguido de acréscimo até a altura de inserção do primeiro galho vivo. A ocorrência dos menores valores de contração no topo das árvores pode ser atribuída à maior porcentagem de lenho juvenil, além de menores valores de massa específica básica.

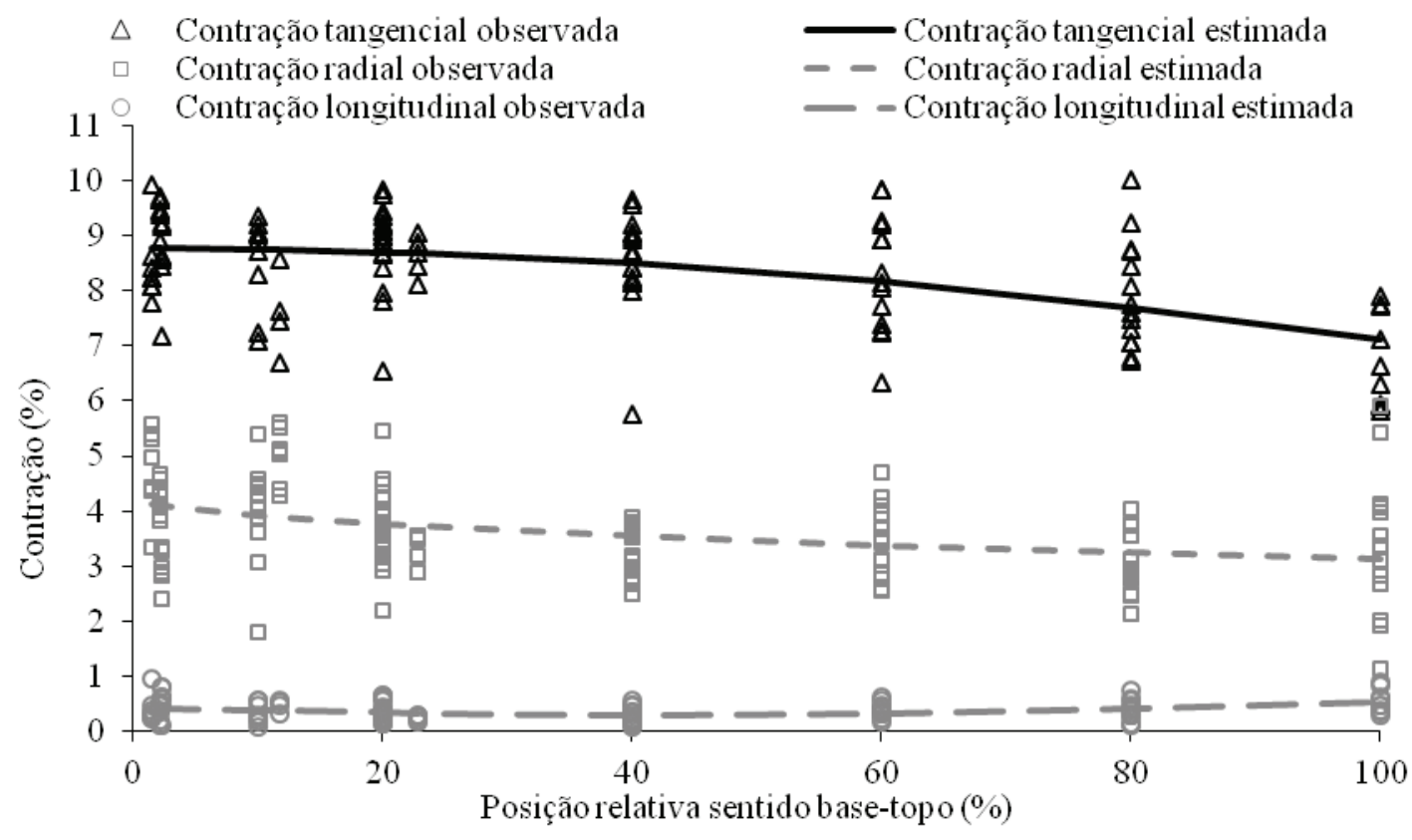

Figura 1. Contração da madeira de Chrysophyllum marginatum nas direções tangencial, radial e longitudinal, em função da posição relativa no sentido base-topo. 
Semelhante ao verificado neste estudo, a maior alteração dimensional da madeira, de acordo com Panshin \& De Zeeuw (1980); Moreira (1999); Oliveira \& Silva (2003) e Vicente et al. (2005), se manifesta no sentido tangencial aos anéis de crescimento, seguido pelo radial e praticamente desprezível no sentido longitudinal. De um modo geral, a pequena variação no sentido longitudinal pode ser explicada pela forma alongada e a organização vertical da maioria dos elementos constituintes do lenho, o que resulta em menor quantidade de paredes celulares, por unidade de comprimento, quando em comparação com as existentes nos sentidos tangencial e radial. A anisotropia transversal tem como preceitos as teorias da restrição oferecida pelos raios; da interação entre lenho outonal e primaveril; da modificação no alinhamento das microfibrilas e da variação na espessura das camadas da parede celular.

O valor médio encontrado para a contração volumétrica(12,4\%) foi classificado como de média retração, pois se situou na faixa entre $12,3 \%$ e 19,4\% (Instituto de Pesquisas Tecológicas, 1985). Scanavaca Junior \& Garcia (2004) relataram que quanto menor essa característica melhor é a madeira para marcenaria, em função da menor movimentação dimensional. Assim, evidenciou-se em função desses resultados que a espécie tem potencial para ser utilizada com maior aproveitamento para construção civil em ambientes internos como, por exemplo, portas internas e rodapés.
$\mathrm{O}$ coeficiente anisotrópico (CA) médio encontrado foi de 2,3 caracterizando a madeira, segundo Durlo \& Marchiori (1992) e Oliveira (1988), como ruim, pois apresenta maior probabilidade de formação de fendas, rachaduras, empenamentos, além de ser de difícil secagem. Assim, para usos que demandem maior estabilidade dimensional, devem ser empregadas algumas técnicas que reduzem o aparecimento de defeitos na madeira associados a essa característica. Entretanto, apesar do elevado valor encontrado para a razão entre a contração tangencial e radial, de acordo com Oliveira \& Silva (2003), a faixa de variação para essa relação é, geralmente, de 1,5 a 2,5.

$\mathrm{O}$ coeficiente anisotrópico em função da posição relativa no sentido base-topo (Figura 2) apresentou valores observados na faixa de 1,4 (base) a 2,8 (topo) com uma tendência de acréscimo entre a base e o DAP seguido de estabilização a partir dessa posição até o topo (cerca de 10\% da altura de inserção do primeiro galho vivo). Esses resultados, de acordo com Bao et al. (2001), são esperados, pois o coeficiente anisotrópico é maior no lenho juvenil (maior porcentagem próximo ao topo) do que no lenho adulto (maior porcentagem próximo à base), o que evidencia a maior instabilidade dimensional do lenho juvenil.

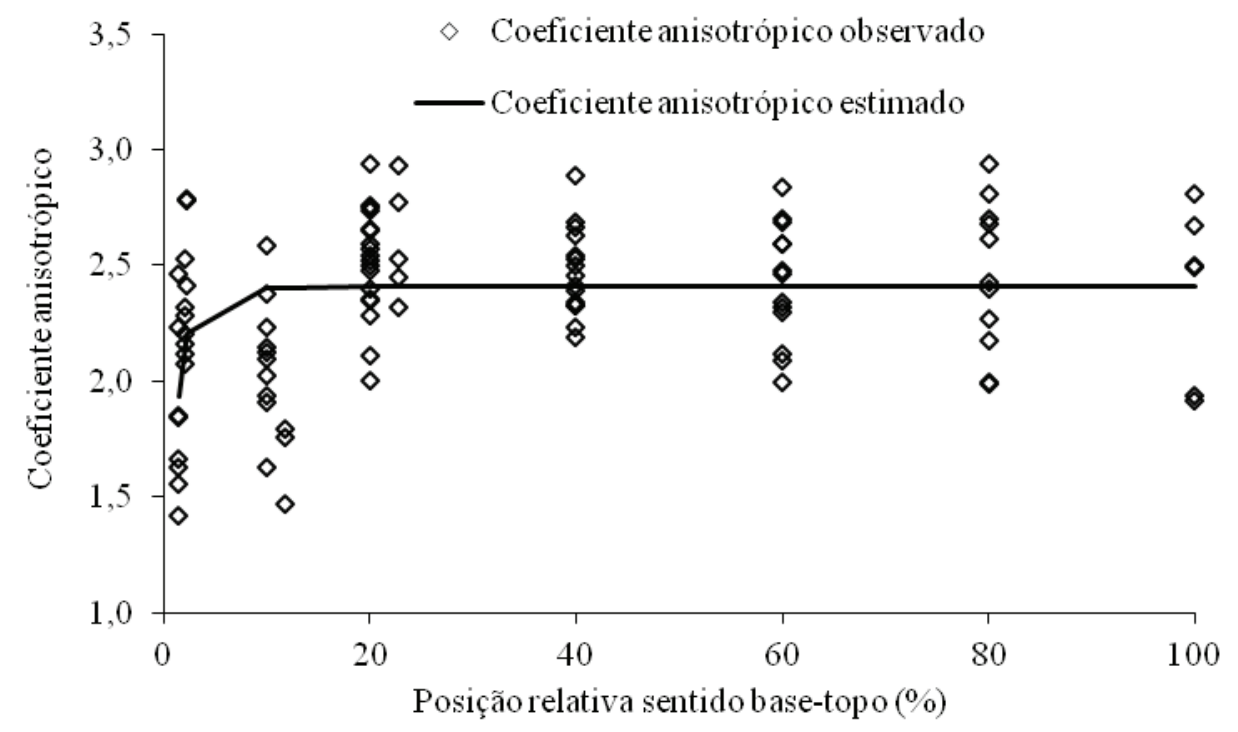

Figura 2. Coeficiente anisotrópico da madeira de Chrysophyllum marginatum em função da posição relativa no sentido axial. 
Com relação aos modelos selecionados para descrever as propriedades físicas de Chrysophyllum marginatum, , observou-se que apenas na contração longitudinal duas variáveis independentes foram selecionadas para reproduzir a variação no sentindo base-topo (Tabela 1).

$\mathrm{Na}$ análise do teor de umidade saturado, observou-se variação inversamente proporcional à massa específica básica, com tendência crescente no sentido base-topo, variando de 78,2 (base) a 130,2\% (topo) (Figura 3), corroborando com os resultados encontrados para Eucalyptus saligna por Lopes \& Garcia (2002) e para Eucalyptus grandis por Lopes (2003). Da mesma forma, Busnardo et al. (1983) e Foelkel et al. (1983) também observaram correlação inversa entre essas duas propriedades.

Tabela 1. Modelos selecionados pelo procedimento estatístico para descrever as propriedades físicas da madeira de Chrysophyllum marginatum.

\begin{tabular}{lcccc}
\hline \multicolumn{1}{c}{ Propriedade } & Modelo & F cal. & $\mathbf{R}_{\text {aj. }}^{2}$ & CV \\
\hline Contração tangencial & $\beta_{\mathrm{tg}}=8,76946-0,00016608 * \mathrm{P}^{2}$ & 40,41 & 0,26 & 10 \\
\hline Contração radial & $\beta_{\mathrm{rd}}=4,26636-0,11514 * \sqrt{\mathrm{P}}$ & 19,95 & 0,13 & 23 \\
\hline Contração longitudinal & $\beta_{\mathrm{lg}}=0,42682+0,00007242 * \mathrm{P}^{2-}$ & 07,61 & 0,10 & 49 \\
\hline Coeficiente anisotrópico & $\mathrm{CA}=2,41164-1,0764 * 1 / \mathrm{P}^{2}$ & 19,38 & 0,16 & 14 \\
\hline Teor de umidade saturado & $\mathrm{TUS}=88,913-2,272 * \sqrt{\mathrm{P}}$ & 26,82 & 0,17 & 14 \\
\hline Massa específica básica & $\mathrm{MEB}=0,063747-0,00792 * \sqrt{\mathrm{P}}$ & 27,80 & 0,17 & 08 \\
\hline
\end{tabular}

Em que: $\beta_{\mathrm{tg}}, \beta_{\mathrm{rd}},{ }_{\mathrm{g}}=$ contração tangencial, radial e, longitudinal, respectivamente (\%); $\mathrm{CA}=$ coeficiente anisotrópico; TUS = teor de umidade saturado (\%); $\mathrm{MEB}=$ massa específica básica $\left(\mathrm{kg} \mathrm{m}^{-3}\right) ; \mathrm{P}=$ posição relativa no sentido axial; $\mathrm{F}_{\text {cal. }}=$ valor de $\mathrm{F}$ calculado; $\mathrm{R}_{\text {aj. }}^{2}=$ coeficiente de determinação ajustado; $\mathrm{CV}=$ coeficiente de variação (\%).

$\square \quad$ Teor de umidade saturado observado — - Teor de umidade saturado estimado

- Massa especifica básica estimada $\triangle$ Massa específica básica observada

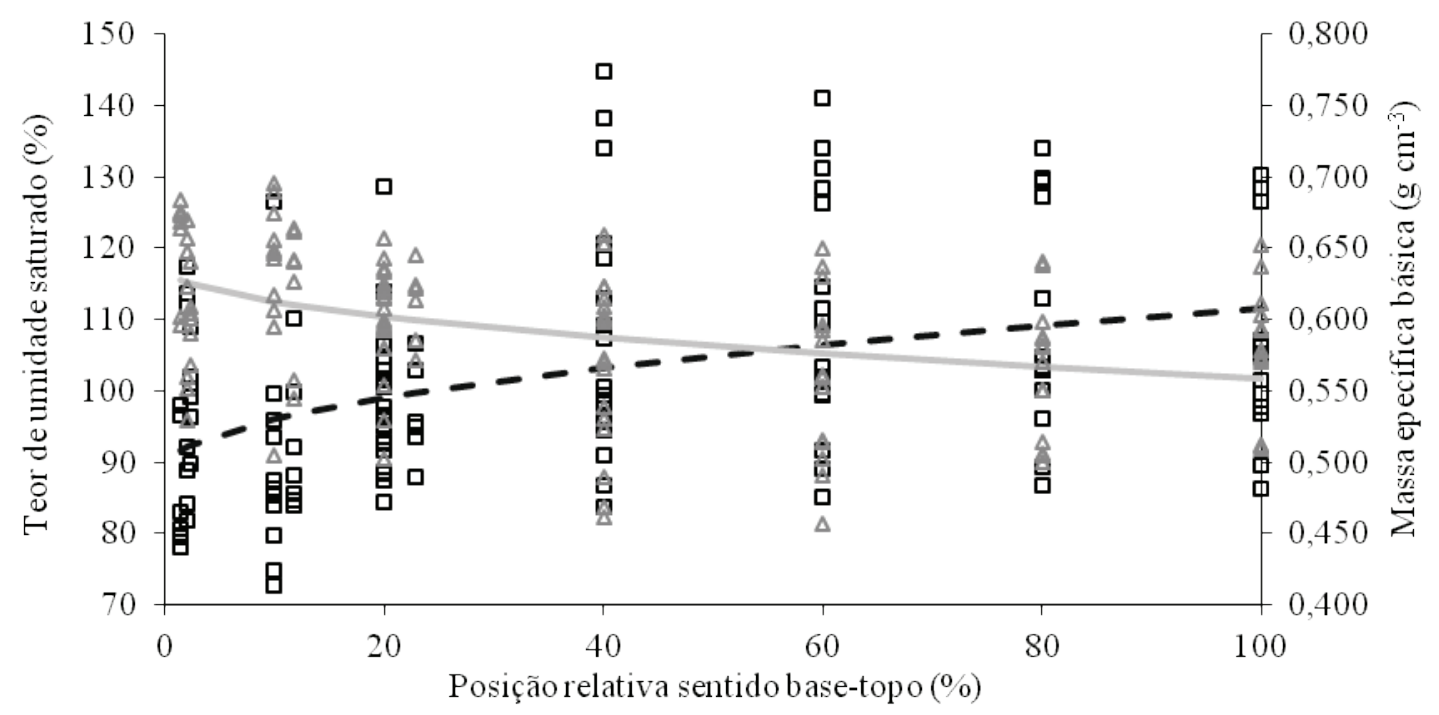

Figura 3. Teor de umidade saturado e massa específica básica da madeira de Chrysophyllum marginatum em função da posição relativa no sentido axial. 
A massa específica básica apresentou tendência decrescente no sentido base-topo em virtude da porção superior das árvores, possivelmente, por apresentarem maior porcentagem de lenho juvenil do que lenho adulto, resultando em mais espaços vazios e diminuindo a quantidade de material lenhoso por unidade de volume.

A média aritmética da massa específica básica foi $594 \mathrm{~kg} \mathrm{~m}^{-3}$, classificada como média, segundo Marques et al. (1997). Diferentemente dessa descrição, Lorenzi (2002) caracterizou a espécie como pesada $\left(780 \mathrm{~kg} \mathrm{~m}^{-3}\right)$.
Essa diferença, segundo Tomazello Filho (1985); Silva et al. (2004) e Alzate et al. (2005), pode ser atribuída a diferenças de idade, fatores ambientais, genéticos ou ainda, na forma de obtenção dos valores, ora aritméticos, ora ponderados.

\section{Flexão estática}

Na Tabela 2 estão representados os dados da análise de resistência à flexão estática nas condições seca ao ar e úmida.

Tabela 2. Dados referentes à flexão estática com análise da variância e teste de comparação de médias para de Chrysophyllum marginatum.

\begin{tabular}{cccccc}
\hline \multirow{2}{*}{ Variável } & \multicolumn{2}{c}{ Condição } & $\begin{array}{c}\text { Razão } \\
(\mathbf{x} / \mathbf{y})\end{array}$ & $\mathbf{F}_{\text {cal. }}$ & Prob. $>$ F \\
\cline { 2 - 3 } & Seca ao ar $(\mathbf{x})$ & Úmida $(\mathbf{y})$ & 1,39 & 6,45 & $0,017^{*}$ \\
\hline $\mathrm{F}_{\text {máx. }}(\mathrm{N})$ & $\begin{array}{c}2311 \mathrm{a} \\
( \pm 761)^{1}\end{array}$ & $\begin{array}{c}1663 \mathrm{~b} \\
( \pm 566)\end{array}$ & & & $0,004^{* *}$ \\
MOR $\left(\mathrm{N} \mathrm{m}^{-2}\right)$ & $\begin{array}{c}84564752 \mathrm{a} \\
( \pm 27751910)\end{array}$ & $\begin{array}{c}55627895 \mathrm{~b} \\
( \pm 19596517)\end{array}$ & 1,52 & 10,08 & $0,014^{*}$ \\
MOE $\left(\mathrm{N} \mathrm{m}^{-2}\right)$ & $\begin{array}{c}10219833303 \mathrm{a} \\
( \pm 2073399742)\end{array}$ & $\begin{array}{c}7732465071 \mathrm{~b} \\
( \pm 2674483219)\end{array}$ & 1,32 & 6,99 & $0,023^{*}$ \\
TLP $\left(\mathrm{N} \mathrm{m}^{-2}\right)$ & $\begin{array}{c}47403333 \mathrm{a} \\
( \pm 18225590)\end{array}$ & $\begin{array}{c}32779120 \mathrm{~b} \\
( \pm 13156211)\end{array}$ & 1,45 & 5,86 & \\
\hline
\end{tabular}

Em que: $\mathrm{F}_{\text {max. }}=$ carga máxima aplicada; $\mathrm{MOR}=$ módulo de ruptura; $\mathrm{MOE}=$ módulo de elasticidade; $\mathrm{TLP}=$ tensão no limite proporcional; $\mathrm{F}_{\text {cal. }}=$ valor de $\mathrm{F}$ calculado; Prob. $>\mathrm{F}=$ nível de probabilidade de erro. ${ }^{1}=$ Desvio padrão entre as médias; $* \mathrm{e}^{* *}$ $=$ significativo ao nível de $5 \%$ e $1 \%$ de probabilidade de erro, respectivamente. Médias com mesma letra na linha não diferem estatisticamente pelo teste de Tukey com confiabilidade de $95 \%$ nas condições seca ao ar (x) e úmida (y);

A comparação das características mecânicas relacionadas com o ensaio de flexão estática de F máx., MOR, MOE e TLP, em duas condições de umidade, revelou diferença entre as médias obtidas (Tabela 2) e, como esperado, os maiores valores foram encontrados na condição seca ao ar. Segundo Kollmann \& Côté (1968), o aumento da resistência da madeira, com o decréscimo no teor de umidade pode ser considerado um resultado tanto do fortalecimento e rigidez dos elementos estruturais da madeira, como da sua compactação, devido à contração que acompanha a perda de água.

Os valores da razão encontrada para o teste de flexão estática, tanto para o $\operatorname{MOE}(1,32)$ como para o MOR $(1,52)$, se aproximaram dos encontrados por Haselein et al. (2002) para Eucalyptus saligna, que foram iguais a 1,29 e 1,40, respectivamente. Cabe ressaltar que os valores de MOE e de MOR citados na Tabela 2 foram próximos aos relatados por esses autores, com valores de $9267284250 \mathrm{~N} \mathrm{~m}^{-2}$ e $72078877 \mathrm{~N} \mathrm{~m}^{-2}$, respectivamente. Da mesma forma, os resultados também estão nos limites citados por Bodig \& Jayne (1982) para a razão madeira seca e verde de folhosas, variando de 1,11 até 1,53 para MOE e de 1,32 até 2,10 para MOR.
Stangerlin et al. (2010), estudando as propriedades de flexão estática de Carya illinoinensis (Wangenh.) K. Koch, evidenciaram que os módulos de elasticidade e ruptura, com valores semelhantes aos descritos na Tabela 2 e iguais a $10.101 .634 .032 \mathrm{~N} \mathrm{~m}^{-2}$ e 82.964.259 $\mathrm{N} \mathrm{m}^{-2}$, respectivamente, apresentaram tendência de redução com acréscimo de umidade o que, de acordo com Lahr (1999), modifica substancialmente os valores de resistência mecânica. Assim, para cada tipo de esforço mecânico há uma diminuição da resistência com o aumento do teor de umidade, até que o ponto de saturação das fibras seja atingido (Freitas, 1982).

\section{Conclusões}

O padrão de variação da contração tangencial, da contração radial e da massa específica básica de Chrysophyllum marginatum apresentou tendência de decréscimo no sentido base-topo, com valores máximos na altura de corte e mínimos próximos à inserção do primeiro galho vivo. 
A contração longitudinal apresentou tendência de decréscimo até, aproximadamente, $40 \%$ da altura, seguido de acréscimo até a inserção do primeiro galho vivo.

Diferentemente das demais propriedades físicas testadas, o teor de umidade saturado apresentou uma variação crescente no sentido longitudinal das árvores.

$\mathrm{O}$ coeficiente anisotrópico foi considerado ruim e, portanto, com restrições para usos que demandem estabilidade dimensional. Dessa forma, a espécie pode ser utilizada com maior aproveitamento para construção civil e para produção de lenha e carvão.

A condição de umidade seca ao ar apresentou maior resistência para flexão estática do que a saturada e os valores de módulo de elasticidade e de ruptura foram semelhantes aos encontrados em estudos de Eucalyptus saligna e Carya illinoinensis.

\section{Referências}

Alzate, S. B. A. et al. Variação longitudinal da densidade básica da madeira de clones de Eucalyptus grandis Hill ex. Maiden, E. saligna Sm. e E. grandis x urophylla. Scientia Forestalis, n. 68, p. 87-95, 2005.

American Society for Testing and Materials. ASTM D 143-94: standard test methods for small clear specimens of timber. Philadelphia, 1995.

Bao, F. C. et al. Differences in wood properties between juvenile wood and mature wood in 10 species grown in China. Wood Science and Technology, v. 35, n. 4, p. 363-375, 2001. DOI: 10.1007/s002260100099.

Bodig, J. \& Jayne, B. Mechanics of wood and wood composites. Nova York: Van Nostrand Reinhold, 1982. 712 p.

Brasil, M. A. M. \& Ferreira, M. Variação da densidade básica e das características das fibras em Eucalyptus grandis Hill ex Maiden ao nível do DAP: análise preliminar. IPEF, n. 5, p. 81 - 90, 1972.

Burger, L. M. \& Richter, H. G. Anatomia da madeira. São Paulo: Nobel, 1991. $154 \mathrm{p}$.

Busnardo, C. A. et al. Umidade ao abate da madeira e da casca de Eucalyptus grandis. Silvicultura, v. 8, n. 28, p. $749-753,1983$.

Calderazzo, D. N. \& Yamaji, F. M. Propriedades físicas da madeira de pinus relacionadas à higroscopia. Anais de Eventos da UFSCar, v. 6, n. 3, 2010.

Durlo, M. A. \& Marchiori, J. N. C. Tecnologia da madeira: retratibilidade. Santa Maria, RS: CEPEF/FATEC, 1992. 33 p. (Série técnica, 10).

Freitas, A. Potencial de utilização de madeiras em construções. In: ENCONTRO BRASILEIRO EM PRESERVAÇÃO DE MADEIRAS, 1982, São Paulo. Anais... São Paulo: IPT, 1982.

Foelkel, C. E. B. et al. Método do máximo teor de umidade aplicado à determinação de densidade básica da madeira do eucalipto. Silvicultura, v. 8, n. 28, p. $792-796,1983$.

Haselein, C. R. et al. Propriedades de flexão estática da madeira úmida e a $12 \%$ de umidade de um clone de Eucalyptus saligna Smith sob o efeito do espaçamento e da adubação. Ciência Florestal, v. 12, n. 2, p. 147-152, 2002. DOI: $10.5902 / 198050981689$.

Haygreen, J. G. \& Bowyer, J. L. Forest products and wood science: an introduction. Ames: Iowa State University, 1982. 459 p.
IBGE. Mapas temáticos. Rio de Janeiro, [2014]. Disponível em: <http:// www.mapas.ibge.gov.br/tematicos>. Acesso em: 16 jul. 2014.

Instituto de Pesquisas Tecnológicas. Madeira: o que é e como pode ser processada e utilizada. Boletim ABPM, n. 36, p. 1-189, 1985.

Kollmann, F. F. P. \& Côté, W. A. Principles of wood science and technology. Berlim: Springer-Verlag, 1968. v. 1. 592 p.

Lahr, F.A.R. Informações sobre madeira. São Carolos: EESC, 1999. 27 p.

Lima, J. T. et al. Variation in wood density and mechanical properties in Eucalyptus clones. In: THE FUTURE OF EUCALYPTUS FOR WOOD PRODUCTS. 2000, Launceston, Tasmania. Proceedings... Launceston: IUFRO, 2000. p. 282-291.

Lopes, G. de A. \& Garcia, J. N. Densidade básica e umidade natural da madeira de Eucalyptus saligna Smith, de Itatinga, associadas aos padrões de casca apresentados pela população. Scientia Forestalis, n. 62, p. 13-23, 2002.

Lopes, M. C. Agrupamento de árvores matrizes de Eucalyptus grandis em função das variáveis dendrométricas e das características tecnológicas da madeira. 2003. 111 f. Dissertação (Mestrado em Engenharia Florestal) - Universidade Federal de Santa Maria, Santa Maria.

Lorenzi, H. Árvores brasileiras: manual de identificação e cultivo de plantas arbóreas do Brasil. 2. ed. Nova Odessa: Instituto Plantarum, 2002. 384 p.

Marques, M. H. B. et al. Madeiras da Amazônia: características e utilização. Brasília, DF: IBAMA, 1997. 141 p.

Mattos, B. D. et al. Variação axial da densidade básica da madeira de três espécies de gimnospermas. Revista Brasileira de Ciências Agrárias, v. 6, n. 1, p. 121-126, 2011. DOI: 10.5039/agraria.v6i1a1080.

Moreira, W. S. Relações entre propriedades físico-mecânicas e características anatômicas e químicas da madeira. 1999. 107 f. Tese (Doutorado em Ciências Florestais) - Universidade Federal de Viçosa, Viçosa, MG.

Oliveira, J. T. S. Estudo das propriedades físicas e tecnológicas da madeira da pindaíba (Xylopia sericea St. Hill.). 1988. 106 f. Dissertação (Mestrado em Ciências Florestais) - Universidade Federal de Viçosa, Viçosa, MG.

Oliveira, J. T. S. \& Silva, J. C. R. Variação radial da retratibilidade e densidade básica da madeira Eucalyptus saligna Smith. Revista Árvore, v. 27, n. 3, p. 381-385, 2003. DOI: 10.1590/S0100-67622003000300015.

Panshin, A. J. \& de Zeeuw, C. Text book of wood technology. 4th. ed. New York: Mc Graw Hill, 1980. 722 p.

Rezende, M. A. et al. Estudo das inter-relações entre massa específica, retratibilidade e umidade da madeira do Pinus caribaea var. hondurensis aos 8 anos de idade. IPEF, n. 48/49, p. 133-141, 1995.

Rezende, M. A. Retratibilidade da Madeira de Pinus Caribaea var. hondurensis e de Eucalyptus grandis e suas Relações com a umidade e densidade. Scientia Forestalis, Piracicaba. n. 64, p. 120-127, 2003.

Roque, R. A. M. Variação da anatomia e da densidade básica da madeira de Gmelina arbórea (Roxb.), em diferentes condições de clima e de manejo na Costa Rica. 2005. 181 f. Tese (Doutorado em Recursos Florestais) Universidade de São Paulo, Piracicaba.

Scanavaca Junior, L. \& Garcia, J. N. Determinação das propriedades físicas e mecânicas da madeira de Eucalyptus urophylla. Scientia Forestalis, n. 65, p. $120-129.2004$.

Serpa, P. N. et al. Avaliação de algumas propriedades da madeira de Eucalyptus grandis, Eucalyptus saligna e Pinus elliottii. Revista Árvore, v. 27, n. 5, p. $723-33$, 2003. DOI: 10.1590/S0100-67622003000500015.

Silva, J. C. et al. Influência da idade e da posição radial na massa específica básica na madeira de Eucalyptus grandis Hill ex. Maiden. Floresta, v. 34, n. 1 , p. $13-22$, 2004. DOI: $10.5380 /$ rf.v34i1.2371. 
Stangerlin, D. M. et al. Propriedades de flexão estática da madeira de Carya illinoinensis em duas condições de umidade. Ciência da Madeira, v. 1, n. 2, p. 70-79, 2010.

Statistical Analysis System. Programa de computador: ambiente VM. Cary, 1993. Versão 6.08.

Tomazello Filho, M. Variação radial da densidade básica e da estrutura anatômica da madeira de Eucalyptus saligna e Eucalyptus grandis. IPEF, v. 29, p. 37-45, 1985.

Trevisan, R. et al. Efeitos da intensidade de desbaste nas características dendrométricas e tecnológicas da madeira de Eucalyptus grandis. Ciência Florestal, v. 17, n. 4, p. 377- 87, 2007. DOI: 10.5902/198050981969.
Tsoumis, G. Science and technology of wood: structure, properties and utilization. New York: Van Nastrnd Reinold, 1991. 494 p.

Vicente, A. T. V. et al. Variação radial e axial da massa especifica básica e das contrações da madeira de quatro procedências de Pinus. Brasília, DF: Embrapa Recursos Genéticos e Biotecnologia, 2005.29 p. (Embrapa Recursos Genéticos e Biotecnologia. Boletim de pesquisa e desenvolvimento, 98).

Vital, B. R. Métodos de determinação da densidade da madeira. Viçosa, MG: SIF/UFV, 1984. p. 1-21. (Boletim técnico, 1). 
